\title{
ACCURACY AND SPACE BANDWIDTH IN SPACE VARIANT OPTICAL CORRELATORS
}

\author{
David CASASENT and Demetri PSALTIS \\ Carnegie-Mellon University, Department of Electrical Engineering, Pittsburgh, PA 14213, USA
}

Received 28 June 1977

Revised manuscript received 23 August 1977

\begin{abstract}
Numerous space variant optical pattern recognition systems using coordinate transformations have been reported recently In this paper, we consider the required space bandwidth of these systems and the resultant accuracy of these novel deformation invariant correlators.
\end{abstract}

\section{Introduction}

Space variant optical processing techniques have been investigated for several years [1-3] but only recently have they been applied to pattern recognition systems [4]. A general formulation of space variant correlation using coordinate transformations to produce optical pattern recognition systems invariant to specific input deformations has recently been described [5]. One-dimensional functions are used for simplicity only. The undeformed or reference function $f(x)$ is assumed to be degraded by the deformation function $g(x, a)$ producing a deformed function $f^{\prime}(x)=f\left(x^{\prime}\right)=f[g(x, a)]$ where $a$ is the deformation parameter. When the coordinate transformation $h^{-1}(x)=\xi$ is applied to these functions, two new functions $f_{1}(\xi)$ and $f_{1}^{\prime}(\xi)$ result. The coordinate transformation is chosen such that the new functions are shifted versions of one another $f_{1}(\xi)=f_{1}^{\prime}\left(\xi-\xi_{0}\right)$ where $\xi_{0}$ is a constant and depends only on $a$. These new functions $f_{1}$ and $f_{1}^{\prime}$ are then used in any conventional space invariant optical correlator. The entire system consisting of the coordinate transformation followed by a space invariant correlator becomes a space variant correlator which is invariant to the dis. tortion $g(x, a)$ and in which the location of the output correlation peak is proportional to the distortion parameter $a$. In the following sections, the space bandwidth (SBW) requirements and accuracy are analyzed for various space variant correlators using coordinate transformations. We are concerned with both spatial and amplitude accuracy and discuss how the implementation of the required coordinate transformation and the available system SBW product affects the accuracy of the resultant correlator.

\section{General considerations}

The space bandwidth required to represent the various coordinate distorted functions will be different from that of the undistorted function and will depend on the specific coordinate transformation used; this in turn depends on the specific deformation being considered. If the original input has resolution $\Delta x$, the resolution element of the coordinate deformed function is

$\Delta \xi=\frac{\mathrm{d} h^{-1}(x)}{\mathrm{d} x} \Delta x$.

This resolution varies with $\xi$; but since the space invariant system treats each point along the $\xi$ line identically, its resolution is uniform in $\xi$. The required resolution element along the $\xi$ line is thus

$\Delta \xi \leqslant \min \left[\frac{\mathrm{d} h^{-1}(x)}{\mathrm{d} x}\right] \Delta x$.

We denote the spatial extent of the input function from $\underline{x} \leqslant x \leqslant \bar{x}$ where $x_{\max }=\bar{x}$ and $x_{\min }=\underline{x}$ and for the coordinate transformed function $\underline{\xi} \leqslant \xi \leqslant \bar{\xi}$. The 
bandwidth in $\xi$ space is proportional to $(1 / \Delta \xi)^{-1}$ and the space bandwidth $N^{\prime}$ in $\xi$ space must satisfy

$N^{\prime} \geqslant|c| \frac{\max \left[h^{-1}(x)\right] \cdots \min \left[h^{-1}(x)\right]}{\min \left[\mathrm{d} h^{-1}(x) / \mathrm{d} x\right] \Delta x}$,

where $c$ is a constant. $N^{\prime}$ is expected to be larger than the required space bandwilth $N$ in $x$ space. A space variant system is not shift invariant and this becomes a serious consideration because accurate input recording is required in the implementation of a space variant system.

\section{Mellin transform}

The most well known space variant processor is the Mellin transform [5,6], in which $x^{\prime}=g(x, a)=a x$, and for which $\xi=\ln x$ describes the required coordinate transformation. This space variant system is of concern because it is scale invariant. For this system, $\underline{\xi}=\ldots \infty$. Because $\ln 0$ is not defined, we choose $\underline{x}=\delta$

$=M \Delta x$, where the space bandwidth in the region $0 \leqslant x<\delta$ of the input is $M$. With $\bar{x}=N \Delta x$, we find $\bar{\xi}=\ln (N \Delta x), \Delta \xi=1 / N$ and from eq. (3)

$N^{\prime} \geqslant N \ln (N / M)$.

For this case we note that $M / N$ is the portion of the input lying in $0 \leqslant x \leqslant \delta$ and that the accuracy (proportional to $N / M$ ) and space bandwidth requirements of this system are interrelated. Since the purpose of this system is to effect scale invariant correlation, we note that the scale factor $a$ to be accommodated does not affect $N$ but increases $M$ proportional to $a$. Thus $N^{\prime}$ is determined by $N$, accuracy $(N / M)$ and the desired range of $a$. As the accuracy increases, so does $N^{\prime}$. As $a$ increases, so does $N^{\prime}$. For $N=500,1 \%$ accuracy requires $N^{\prime}=2650$ and the maximum $a$ is $100 \% .2 \%$ accuracy requires $N^{\prime}=2300$. For $a=200 \%$ and $1 \%$ accuracy, we require $N^{\prime}=2850$.

\section{Exponentiated coordinate distortions}

A space variant correlator invariant to distortions of the form $x^{\prime}=g(x, a)=x^{a}$ has recently been reported [7]. Such distortions arise in scanning devices, aberrations, non-uniform target motion, and in imaging from curved surfaces. The required coordinate trans- formation has been shown to be $\xi=\ln (\ln x)$. For this system, we ignore the portion of the function in $0>x \geqslant 1$ and restrict $x$ to $1>x>\infty$. For this case, we find by extension of eq. (4) that

$N^{\prime} \geqslant N(\ln N)(N / M) \ln [(N / M) \ln (N / M)]$

\section{Rotation invariance $[4,8]$}

When two functions $f(x, y)$ and $f^{\prime}(x, y)$ are rotated versions of one another, a 2-D notation must be used. We describe the deformations by

$f^{\prime}(x, y)=f\left[g_{x}\left(x, y, \theta_{0}\right), g_{y}\left(x, y, \theta_{0}\right)\right]$,

where the distortion functions are

$g_{x}\left(x, y, \theta_{0}\right)=\sqrt{x^{2}+y^{2}} \cos \left[\tan ^{-1}(y / x)+\theta_{0}\right]$

$g_{y}\left(x, y, \theta_{0}\right)=\sqrt{x^{2}+y^{2}} \sin \left[\tan ^{-1}(y / x)+\theta_{0}\right]$,

and the distortion parameter is the angle of rotation $\theta_{0}$. For this case, the coordinate transformed functions are $f_{1}(\xi, \eta)$ and $f_{1}^{\prime}(\xi, \eta)$ where two coordinate variables are needed. These required coordinate transformations are:

$\xi=\tan ^{-1}(y / x), \quad \eta=\sqrt{x^{2}+y^{2}}$,

where $(\xi, \eta)=(r, \theta)$, the conventional polar coordinates and

$x=\eta \cos \xi, \quad y=\eta \sin \xi$.

In 2-D, we denote the space bandwidth of the original function by $N^{2}$ and that of the coordinate transformed function by $\left(N^{\prime}\right)^{2}$. With the resolution in the $r$ and $\theta$ coordinates denoted by $\Delta r$ and $\Delta \theta$, we find

$\Delta r=\Delta x, \quad \Delta \theta=\Delta x / \bar{r}=\sqrt{2} / N$ radians,

$(10 a, b)$

where $\bar{r}=r_{\max }=N \Delta x / \sqrt{2}$ and $\bar{\theta}=\theta_{\text {max }}=$ $4 \tan ^{-1}(N \Delta x / 2 \Delta x)=4 \tan ^{-1}(N / 2)$. The bandwidths in $r$ and $\theta$ are $1 / \Delta x$ and $n / \sqrt{2}$ and the 1-D space bandwidths in $r$ and $\theta$ are

$N_{r}^{\prime}=N / \sqrt{2}, \quad N_{\theta}^{\prime}=(4 N / \sqrt{2}) \tan ^{-1}(N / 2)$

from which

$\left(N^{\prime}\right)^{2}=N_{r}^{\prime} N_{\theta}^{\prime}=2 N^{2} \tan ^{-1}(N / 2)=\pi N^{2}$.

The space bandwidth of a polar transformed function must thus be over three times larger than that of the 
associated coordinate transformed functions to maintain the same accuracy.

\section{Implementation accuracy}

Various methods of optically realizing these various space variant optical systems have been suggested. All concern the method by which the input coordinate transformation preprocessing step is implemented. It has been suggested [9], that the coordinate transformation be applied: (a) to the deflection coils of an electron beam addressed spatial light modulator (SLM) [10] by analog modules; (b) or similarly to the acousto-optic deflection cells when using an optically addressed SLM [10]. The Mellin transform has also been implemented using computer generated holograms [11], however this method is not useful when the coordinates are coupled [12] as in the rotation invariant transform and it is of use only with low space bandwid th imagery.

In this section, we compare two non-linear scanning realizations of these transformations in light of the accuracy and space bandwidth analysis of sections 2 to 5 . In image processing, the input pattern is assumed to be sensed by a TV camera. The output video signal is then fed to a real-time SLM (electron beam or laser beam addressed). The output horizontal and vertical ramps from the TV can be modified by analog modules and fed to the deflection circuitry of the SLM. Alternately, the scan of the TV camera itself can be modified. In this latter implementation, a linear scanner san be used for the real-time device. Since $N^{\prime} \gg N$, this consideration becomes vital. When the deflection ignals for the TV are modified, the video signal is clistorted and its time bandwidth is increased by the lactors noted in sections 2 to 5 . The camera and all electronics following it must accomodate this higher time bandwidth. High bandwidth and high slew rate analog modules are needed in such a system. These represent formidable electronic circuits. If the deflection signals of the deflector for the SLM are deformed instead, the video itself is never altered and only the real-time SLM itself need satisfy the large space band. width.

A final consideration in the use of a TV system to realize the required coordinate transformations is un- desired amplitude modulation. In space variant processing, the coordinate transformation should only relocate function values and not alter the value of the function at any point. However, non-linear scanning produces non-uniform modulation over the SLM or the TV's photo-surface. This occurs because all SLM's are energy detectors, with recorded intensity proportional both to the electron beam current and the inverse of the beam's speed. With non-linear scanning, the nonconstancy of the speed of the electron beam over the SLM's surface causes an undesired amplitude modulation necessitating preamplification and techniques similar to gamma correction to compensate for this distortion. Similar remarks hold for optically addressed SLM's.

If $\Delta \xi$ is the size of a resolution element on an electron beam addressed SLM and $P(t)$ the power delivered by the electron beam, the intensity $I\left(\xi_{\mathrm{a}}\right)$ recorded at $\xi=\xi_{\mathrm{a}}$ is

$$
\begin{aligned}
I\left(\xi_{\mathrm{a}}\right) & \approx \int_{\xi_{\mathrm{a}}}^{\xi_{\mathrm{a}}+\Delta \xi} P(t) \mathrm{d} t \approx P\left(\exp \xi_{\mathrm{a}}\right) \int_{\xi_{\mathrm{a}}}^{\xi_{\mathrm{a}}+\Delta \xi}(\exp \xi) \mathrm{d} \xi \\
& \approx P\left(\exp \xi_{\mathrm{a}}\right) \exp \left(\xi_{\mathrm{a}}\right)[\exp (\Delta \xi)-1] \\
& \approx P\left(\exp \xi_{\mathrm{a}}\right) \Delta \xi \exp \left(\xi_{\mathrm{a}}\right),
\end{aligned}
$$

from which the undesired amplitude modulation $\exp \left(\xi_{\mathrm{a}}\right)$ introduced by the coordinate transformation is apparent. If the amplitude of the video signal is multiplied by $1 / t$ (where $t$ is the time corresponding to the horizontal deflection position of the electron beam), this will compensate for this undesired amplitude modulation.

\section{Summary}

The space bandwidth requirements for a space variant correlator are shown to be larger than those of a conventional system by a factor of 2 to 5 depending on the deformation invariant coordinate transformation used. Implementation by non-linear scanning has been shown to produce undesired nonuniform amplitude modulation requiring additional compensation. 


\section{Acknowledgment}

The support of the Ballistic Missile Defense Advanced Technology Center on contract DASG-60-77 C-0034 for this work is gratefully acknowledged.

\section{References}

[1] T.S. Huang, W.F. Schreiber and O.J. Tretiak, Proc. IEEE 59 (1971) 1586.
[2] G.M. Robbins, Ph. D. Thesis, Mass. Inst. Techn. (1970).

[3] A. Sawchuk, J. Opt. Soc. Amer. 64 (1974) 138.

[4] D. Casasent and D. Psaltis, Proc. IEEE 65 (1977) 77.

[5] D. Psaltis and D. Casasent, Appl. Opt. 16 (1977) 2288.

[6] D. Casasent and D. Psaltis, Opt. Commun. 17 (1976) 59.

[7] D. Casasent and D. Psaltis, Opt. Commun. 18 (1977) 307.

[8] D. Casasent and D. Psaltis, Appl. Opt. 15 (1976) 1795.

[9] D. Casasent and D. Psaltis, Opt. Engr. 15 (1976) 258.

[10] D. Casasent, Proc. IEEE 65 (1977) 143.

[11] D. Casasent and C. Szczutkowski, Opt. Commun. 19 (1976) 217.

[12] D. Casasent and C. Szczutkowski, Proc. Soc. Photo Opto Instru. Engrs. 83 (1976) 91. 